\title{
Attributal Standard Establishment For Spot Colors Accomplished Through Digital Proofing
}

\author{
Mr. Abhijit B. Ladgaonkar \\ M.E. Printing Final Year Pune University, India.
}

\begin{abstract}
Digital Proofing is a way of digital printing in which digtal large format printers are used to replace the traditional system of proofing. Digital proofing system is generally an inkjet or color laser printer component which is being used to simulate the traditional proofing or printing process and it is growing remarkably because of high speed, affordable prices and high color gamut. As now a days so many innovations took palce in printer as well as proofing softwares digital proofing is choice for most of the printers. In digtal proofing 3 factors are affecting the color appearance as well as the quality of the proof-Print equipment, Proofing software and Proofing substrate.

As far as concerning Packaging segment for proofing pantone colors reproduction with digital proofer is crucial task. The main purpose of this experiment is to examine the effect of proofing module as well as the substrate on the quality of Pantone proof. The quality of proof is examined by evaluating the colour in terms of $L a^{*} b^{*}$ and color consistency.
\end{abstract}

Keywords: Spot color Proofing,proofing modules, calibration for digital proofing.

\section{Introduction:}

Digital proofing undergoes enormous technical changes from past few years. Where traditional printers. Service beuro's and in-plant operational industries are struggling for ideal color proofing solution which fullfill their requirement of obtaining correct color proof from the proofing solution. Again when Pantone comes into the role this become more complicated. There are many digital technologies are avialable in the market based on color thermal transfer, elctrophoto graphic and inkjet principle[1]. But Ink jet is the most dominant proofing technology used in the industry which has $75 \%$ share and it is still growing. Latest inkjet technologies are capable to print high quality as well as rich color images on variety of substrates. The right proofing software provides you the assurity to give you the right color on the substrate. Today, high-resolution, drop-on-demand inkjet devices have overcome initial resistance to dominate the low-end to midrange proofing marketplace. As the new emerging technology of inkjet (piezo-electric, epson) has most prominent share in proofing market.

The proof color accuracy is highly affected by the proofing software and the substrate being used for proofing.[2][3] As different print houses using different ink technologies with different media It shows differnt result of color reproduction which again makes proofing, a crucial task. Especially for pantones the result will be more unpredictable. In general term proofing is done on the same printing substrate for close prediction of colors reproduction however working with differnt inks and devices will differ the result on the same printing substrate.Good proofing solution also needs aid of color management which integrates with effective Raster Image Processor (RIP) configuration and profiling techniques.[2][5]

When the printing workflow enters in digital era a suaitable and reliable color proofing solution should be investigated to meet the needs of color printing special concern with pantones as it is the most critical issue in packaging industry. The main purpose of this experiment is to study the effect of the software and the proofing substrate on proof quality. The color reproduction is evaluated in terms of color difference (Delta E 1976) and color consistency. The $\mathrm{x}$-rite Spectro eye is a valuabe tool to evaluate color in terms of Delta E.

\section{Experimental:}

Two types of custom proofing papers were tested (Semigloss art proofing paper 140 GSM and 100 GSM) were tested and compared with two commercially available proofing softwares (Efi XF and Staproof). Different combination of substrate and proofing module were tested with Epson 7900 proofer with HDR inks. ICC profiles are generated for two substrates with two proofing modules with Profile maker. The device was profiled as CMYK device with ECI2002R chart from different modules and calibration setups are created for Epson 7900 with Efi XF and Starproof. A customised Pantone test form were printed on RYOBI OFFSET (6 color). And generated pantone values were loaded into proofing modules to achive the target $\mathrm{L}^{*} \mathrm{a} \mathrm{b}^{*}$ on proofing substrate. Also a customised test form of only pantones (1505 C \& 323C) were printed to evaluate the pantone reproduction in better way. Also some visusl targets and pantone job files were added in the test form to evaluate the effect of pantone overprinting and how proofing module interprets that while proofing. 


\section{Results \& Discussion:}

In this experiment 4 proofing combinations were tested to match offset printing. The paper matching target is 170 GSM Art Paper. The color gamut, Color matching capacity and process consistency for offset printing were tested and compared. The color gamuts of different print combinations were tested using colorthink Pro.3.0 while color differences (Delta E 1976) were measured with x-rite Spectrophotometer. Minitab 16 a statistical software package is used for process consistency analysis.

\section{Gamut Comparison:}

Before doing the pantone color matching analysis gamut comaprison for diffrent print combinations used must be compared. Figure 1 and figure 2 illustrates the digital proofer gamut comparison for 140 GSM and 100 GSM semigloss proofing paper with different proofing modules.

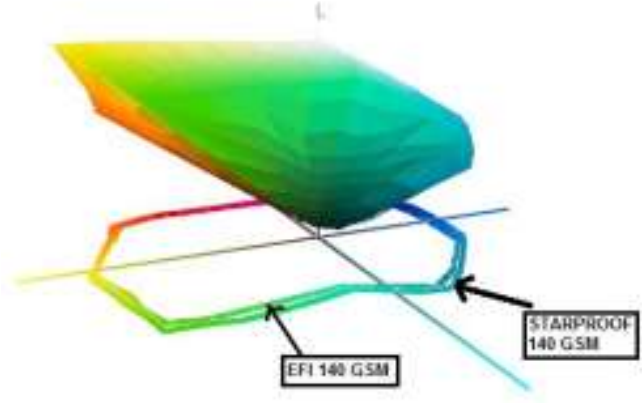

Fig.1- Gamut for 140 GSM Paper

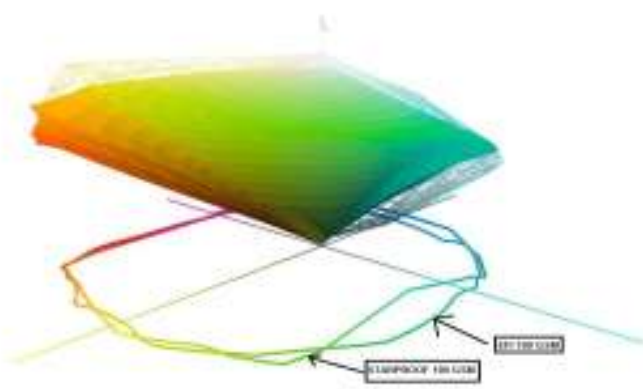

Fig.2 Gamut for 100 GSM Paper

Figure. 1 shows the gamut plotting for 140 GSM Semigloss proofing paper which clearly shows that Starproof has a wider gamut as compare to EFI XF in green and blue region. Figure 2 also explains gamuts of 100 GSM Semigloss proofing paper in which it shows that EFI XF got larger gamut in blues but in green and red area Starproof has the larger gamuts.

Below table:1 shows you the gamut volume we got for diffrent print combinations with epson 7900 proofer. Below table shows that Starproof with 140 Gsm semigloss Paper gives you larger gamut than any other combination, as Efi XF got the second larger gamut.

Table:1 Gamut volumes for differnt proofing substrates with different print combinations

\begin{tabular}{|c|c|c|}
\hline Proofing Module & Substrate & Gamut Volume \\
\hline Starproof & 100 Gsm Semigloss & 608411 color cubic space \\
\hline & 140 Gsm Semigloss & 775499 color cubic space \\
\hline Efi XF & 100 Gsm Semigloss & 500149 color cubicspace \\
\hline & 140 Gsm Semigloss & 683041 color cubic space \\
\hline Offset Pantone Profile & 170 Gsm Art Paper & 440698 color cubic space \\
\hline
\end{tabular}

Pantone Color Matching Capaility:

The quality of proof press is evaluated in terms of Delta E 1976 for both Pantones. Which shows that Starproof +140 GSM semigloss proofing paper has lowest delta e values for both the pantones. The average Delta E for Pantone $368 \mathrm{C}$ is 0.32 and for Pantone $1505 \mathrm{C}$ it is 2.02. As EFI XF with 100 GSM semigloss proofing paper has larger standard Deviation value. The 140 GSM Semigloss proofing paper has good color reproduction capability than 100 GSM. The maximum Delta e 1976 values of 4 print combinations are in range of 0.16-4.5 for Pantone $368 \mathrm{C}$ and 1.21-9.19 for Pantone $1505 \mathrm{C}$. Table: 2 shows the color matching result with different print combinations. With delta e values. 
Table:2 Delta E readings for differnt print combinations

\begin{tabular}{|l|c|c|c|c|c|c|}
\hline Print Combination & & $\begin{array}{l}\text { Delta E } \\
\text { P 368 C }\end{array}$ & & & $\begin{array}{l}\text { Delta E } \\
\text { P 1505 C }\end{array}$ & \\
\hline & Min & Max & Avg. & Min & Max & Avg \\
\hline Starproof + 140 GSM & 0.16 & 1.03 & 0.37 & 1.21 & 2.4 & 2.02 \\
\hline EFI XF + 140 GSM & 3.6 & 5.61 & 4.5 & 4.93 & 5.7 & 5.35 \\
\hline Starproof + 100 GSM & 3.56 & 4.65 & 4.08 & 4.76 & 5.01 & 4.89 \\
\hline Efi XF + 100 GSM & 8.86 & 9.23 & 8.95 & 8.42 & 9.19 & 8.86 \\
\hline
\end{tabular}

Below figures (Fig.3, Fig.4) shows the diagramatic representation of Standard deviation values for differnt print combinations.

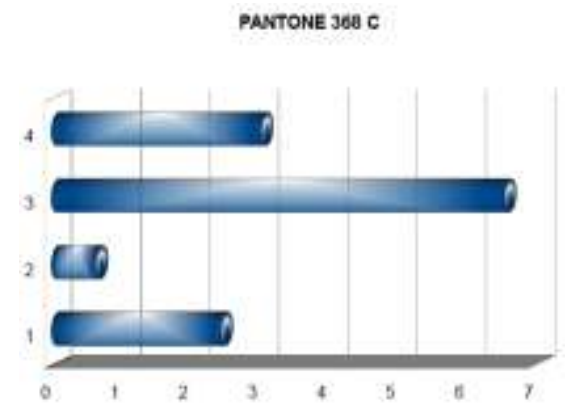

Fig.3 Delta E for Pantone 368 C

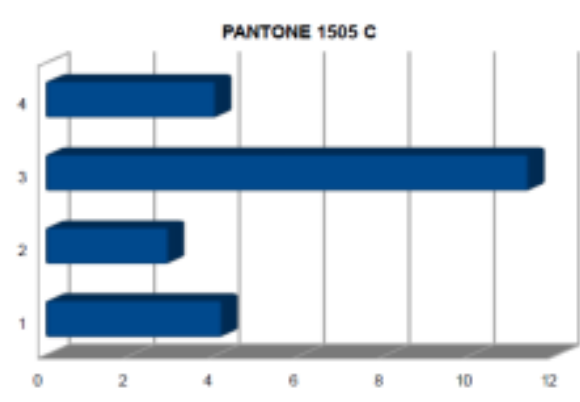

Fig.4 Delta E for Pantone 1505 C

\section{Conclusions:}

In terms of color gamut 140 GSM Semigloss Proofing Paper is having larger gamut than that of 100 GSM with both proofing modules. But when we compare proofing modules Starproof has the larger gamut as compare to EFI. Efi and Starproof is good to repeoduce $1505 \mathrm{C}$ as in 368C Starproof is good. Overall consideration of technical as well as visual results showing that Starproof is having capability to produce better spot colors than EFI though they have larger gamut than Offset.

Selected spot colors showing different behaviour with different combinations used. As Pantone $368 \mathrm{C}$ is hardest color to reproduce which having higher average delta e during whole experiment and $1505 \mathrm{C}$ is easy to reproduce as it is having lesser average delta e. This experiment shows that In digital proofing with custom proofing media color reproduction is mainly affected by proofing module. For implementing a better proofing solution in packaging industry this experiment will work as a guideline. The result is showing that without using manufacturer recommended proofing papers you can achive the results with appropriate module which reduces the costing of your proof. Cosistent Proof quality for spot colors is only achieved by standerdising a proofing process as a whole. Proofer calibration is needed to bring the proofer into a standard condition for a predefined tonal response is guaranteed besides taking care of ink limitation and linearization. The proofer verification module verifies if the proofer output still complies with the standard condition and verifies the consistency of color management with the means of printing media wedge and read by verification module.

\section{Acknowledgement:}

The authors thank Bodhi Professional Solutions Pvt. Ltd. and Sudhindra Offset Pvt Ltd., Banglore for donating all needed infrastructure and resources.

\section{References:}

[1] "Handbook of Print Media", H. Kipphan ISBN: 3-540-67326-1 Springer-Verlag Berlin Heidelberg, 2001.

[2] D. Toth, "Proofing makes the workflow flow", Graphic Arts Monthly, vol. 73, no. 8, pp30-36,2001.

[3] S. Bandyopadhyay, "Effect of paper properties on print quality of inkjet printer", Proceedings of the IS\&T NIP17: International Conference on Digital Printing Technologies, Fort Lauderdale, pp. 141-143. (2001).

[4] E. Hrehorova, A. Sharma, P. D. Fleming, "Color Reproduction Studies in RGB and CMYK Workflows using Inkjet Printer Drivers and RIPs", Proceedings of the $58^{\text {th }}$ TAGA Annual Technical Conference, Vancouver, British Columbia, (2006)

[5] "Understanding Colour Management," (Thomson Delmar Learning, NY). Suchy, Miro, Fleming III, Paul D. and Sharma Abhay. 